\title{
Das zweite Rettungspaket für Griechenland und Perspektiven für die Europäische Zentralbank
}

\author{
Ansgar Belke und Christian Dreger*
}

\section{Die Euro-Gipfelbeschlüsse vom 21. Juli 2011: Hintergründe und Details}

Die europäischen Hilfsmaßnahmen für Griechenland haben im letzten Jahr eingesetzt, nachdem die Ratingagenturen die Kreditwürdigkeit des Staates mehrfach herabgestuft hatten. Damit wurde den Marktteilnehmern ein hohes Ausfallrisiko für Kredite und Staatsanleihen signalisiert. In der Folge erhöhten sich die Kreditzinsen für Griechenland drastisch und die Kurse für Staatsanleihen brachen ein, weil ein Zahlungsausfall nicht mehr ausgeschlossen werden konnte. Im Frühjahr 2010 wurde von der Troika aus Europäischer Union, Europäischer Zentralbank und Internationalem Währungsfonds ein erstes Rettungspaket geschnürt. Der griechischen Regierung sind darin Kreditzusagen im Umfang von 110 Milliarden Euro gemacht worden. Der Internationale Währungsfonds übernimmt 30 Milliarden Euro, die Mitgliedstaaten der Eurozone (ohne Griechenland) 80 Milliarden Euro in Form von bilateralen Kreditzusagen. Die einzelnen Staaten haften in Höhe ihrer Beteiligung am Kapital der Europäischen Zentralbank, das sind 27,9 Prozent für Deutschland. Das Paket ist auf drei Jahre angelegt. Im Gegenzug sollte der griechische Schuldenhaushalt konsolidiert und das Haushaltsdefizit bis 2013 unter die Obergrenze des Stabilitäts- und Wachstumspaktes von 3 Prozent gedrückt werden.

Allerdings hat man die Probleme deutlich unterschätzt. So wurde eine rasche wirtschaftliche Erholung Griechenlands unterstellt, die nicht eingetreten ist und zunehmend unwahrscheinlicher wird. Daher ist im Juli 2011 ein zweites Rettungspaket beschlossen worden. Es läuft bis 2014 und umfasst ein Volumen von 109 Milliarden Euro, die von der EFSF (European Financial Stability Facility) und dem Internationalen Währungsfonds ausgezahlt werden. Indem die Laufzeiten von Krediten verlängert und Zinsen gesenkt werden, verbessern sich die Rückzahlungsmodalitäten für Griechenland. Dies gilt auch für Kredite aus dem ersten Rettungspaket. Des Weiteren ist eine freiwillige Beteiligung privater Anleger vereinbart worden. Darüber hinaus werden die Kompetenzen des EFSF gestärkt. Der Notfallfonds kann nun auch präventiv Staatsanleihen potenziell gefährdeter Staaten am Kapitalmarkt aufkaufen. Ferner ist ein Wiederaufbauplan für Griechenland angekündigt worden, um das wirtschaftliche Wachstum in dem Staat zu stimulieren. Zum Zeitpunkt der Beschlüsse hielten die deutschen Banken Forderungen von über 24 Milliarden Euro an Griechenland. Dabei ist der öffentliche Sektor mit rund 75 Prozent der größte Schuldner. Auf griechische Banken entfällt nur ein Bruchteil (fünf Prozent), der Rest betrifft die Unternehmen des Nichtbankensektors. ${ }^{1}$

* Prof. Dr. Ansgar Belke, Universität Duisburg-Essen und Deutsches Institut für Wirtschaftsforschung (DIW), Berlin.

Prof. Dr. Christian Dreger, Europa-Universität Viadrina Frankfurt an der Oder und Deutsches Institut für Wirtschaftsforschung (DIW), Berlin.

1 Für die folgenden Ausführungen siehe auch Ansgar Belke/Christian Dreger: Das zweite Rettungspaket für Griechenland, in: Wirtschaftsdienst 9/2011, S. 601-607; Ansgar Belke/Christian Dreger: Die vertane Chance der Euro-Retter, in: Handelsblatt, 22.07.2011. 
Von den 109 Milliarden Euro kommen 54 Milliarden Euro Griechenland direkt zugute. Die restlichen 55 Milliarden Euro werden zur Absicherung von Gläubigerbeteiligungen benötigt. Das Paket umfasst vier Bestandteile: 34 Milliarden Euro zur Deckung des Refinanzierungsbedarfs Griechenlands, 20 Milliarden Euro zur Rekapitalisierung griechischer Banken, 35 Milliarden Euro für die Absicherung der Risiken aus der Beteiligung privater Gläubiger und 20 Milliarden gewissermaßen als Anreiz für private Gläubiger, einem freiwilligen Schuldenrückkauf zu schlechteren Bedingungen zuzustimmen. ${ }^{2}$

Die Umschuldung privat gehaltener und bis 2020 fällig werdender Anleihen im Umfang von ungefähr 135 Milliarden Euro soll im Herbst 2011 erfolgen. Das entspricht etwa 90 Prozent der bis 2020 fällig werdenden Anleihen, also 150 Milliarden Euro. Dies nimmt zwar für zehn Jahre den Druck der Finanzmärkte von Griechenland, führt aber erstmals zu einer Einstufung eines Mitgliedstaats der Eurozone (Euro-Staat) als zahlungsunfähig. Denn das Programm wird von den Ratingagenturen gemäß den Vereinbarungen als teilweiser Zahlungsausfall gewertet. Der Zahlungsausfall soll voraussichtlich im Herbst 2011 eingeleitet werden und nur wenige Tage andauern.

Die Europäische Zentralbank sitzt mit im Boot. Die EFSF nimmt der Zentralbank die Risiken eines teilweisen Zahlungsausfalls (, selective default') ab. Denn die Europäische Zentralbank konnte die Übernahme von Bürgschaften für Sicherheiten griechischer Banken in Höhe von bis zu 35 Milliarden Euro durchsetzen. Die EFSF muss sich diese Sicherheiten wiederum am Markt beschaffen. Dies dürfte überwiegend über kurzfristige Geldmarktgeschäfte erfolgen, da der Zeitraum des , selective default ' Griechenlands möglichst kurz ausfallen soll. ${ }^{3}$ Wegen der Überdeckung der Ausleihekapazität der EFSF (,overborrowing requirement'), die für die Sicherung ihres AAA-Ratings erforderlich ist, müssen allerdings mehr als 35 Milliarden Euro aufgenommen werden. Der zusätzliche Betrag dürfte sich auf 12 Milliarden Euro belaufen. Die Sicherheiten für den Anleihenumtausch in Höhe von 135 Milliarden Euro sind bereits am Anfang bereitzustellen. ${ }^{4}$

Zudem sind für Griechenland eine Reihe von Erleichterungen für den Schuldendienst beschlossen worden, die auch für Irland und Portugal übernommen werden. Danach werden die Laufzeiten der Kredite bei günstigeren Zinsen verlängert. Für die Kredite aus dem Notfallfonds EFSF steigt die Laufzeit von 7,5 auf 15 Jahre, möglicherweise auch bis 30 Jahre, und der Zinssatz wird von 4,5 auf 3,5 Prozent sinken. Griechenland soll zehn Jahre lang keine Tilgung leisten müssen. Die schon laufenden bilateralen Kredite der Euro-Staaten werden an die weicheren Zins- und Laufzeitbedingungen angepasst.

\section{Beteiligung des Privatsektors}

Der Privatsektor ist am Rettungspaket beteiligt, aber nur auf freiwilliger Basis. Je nach Präferenz erfolgt dies im Rahmen eines Anleiheverkaufs oder einer Verlängerung fälliger Wertpapiere. ${ }^{5}$ An der Vereinbarung waren 30 private Banken und Versicherungen beteiligt, die 90 Prozent der Gläubiger abdecken. Die EFSF kauft den privaten Gläubigern griechische Anleihen zum Marktpreis mit einem Abschlag ab, wofür sie 20 Milliarden Euro aufbringen

2 Vgl. Belke/Dreger: Das zweite Rettungspaket, 2011.

3 Eventuell wird der Bedarf an Sicherheitsbereitstellung für die Europäische Zentralbank auch mit der Einbeziehung des Privatsektors kombiniert.

4 Charles Forelle: Bailout Will Add to Greek Debt, in: The Wall Street Journal Weblog: Real Time Brussels, 26.07.2011, abrufbar unter: http://blogs.wsj.com/brussels/2011/07/26/bailout-will-add-to-greek-debt/?mod= WSJBlog\&mod=brussels (letzter Zugriff: 05.10.2011).

5 Für die folgenden Ausführungen vgl. Belke/Dreger: Das zweite Rettungspaket, 2011; Belke/Dreger: Die vertane Chance, 2011. 
muss. Nach Berechnungen der Gipfelteilnehmer werden die Privatinvestoren bei dieser Aktion 12,6 Milliarden Euro verlieren. Zudem haben sich die Banken bereit erklärt, fällige Anleihen in neue mit sehr langer Laufzeit und erstklassiger Sicherheit umzutauschen. Bis zum Jahr 2014 wird ein zusätzlicher Abschreibungsbedarf von 37 Milliarden Euro erwartet. Da die Anleihen zum Nennwert in den Büchern stehen, sinkt die Staatsschuld für Griechenland ab der Übernahme durch die EFSF um 12 Prozent des Bruttoinlandsprodukts. Die Banken sollen im Schnitt Abschreibungen von insgesamt 21 Prozent vornehmen. Das ist relativ günstig, weil auf den Märkten Abschläge von bis zu 45 Prozent verlangt werden. So haben die Banken Verluste in dieser Größenordnung schon längst abgeschrieben und können die Wertberichtigungen aufgrund der Beschlüsse zu großen Teilen wieder nach oben korrigieren. Die Banken haben auch die Finanzierung der griechischen Banken längst an die Europäische Zentralbank und damit an den Steuerzahler weitergegeben. Sie profitieren überproportional stark vom Zinsspread griechischer Anleihen, der weit über das Äquivalent eines Schuldenschnitts von 21 Prozent hinausgeht und werden zudem enorm an der technischen Durchführung der Umschuldung verdienen.

\section{Großzügige Erweiterung der EFSF-Kompetenzen bei unzureichendem Schulden- schnitt}

Die Kompetenzen und Interventionsmöglichkeiten der EFSF werden durch die Beschlüsse erheblich ausgeweitet. Der Fonds kann nun im Rahmen einer flexiblen Kreditlinie unter strikten Auflagen auf dem Sekundärmarkt präventiv tätig werden und Anleihen aufkaufen; dies ist vor allem auf Spanien und Italien gemünzt. Für ein solches Engagement müssen einige Voraussetzungen erfüllt sein. Erforderlich sind die Feststellung außergewöhnlicher Umstände durch die Europäische Zentralbank, ein einstimmiger Beschluss der Finanzminister der Euro-Staaten und die Erfüllung von Programmen und Auflagen. Damit soll sichergestellt werden, dass der Fonds nur in Ausnahmefällen von seinen gewachsenen Kompetenzen wirklich Gebrauch macht. Schließlich soll die EFSF den Bankensektor eines betroffenen Staates indirekt durch spezifische Kredite an die jeweilige Regierung stützen können.

Sofern die Spielräume tatsächlich genutzt und auch Anleihen von Staaten aufgekauft werden, die nicht in EFSF-Programmen stecken und wie Italien und Spanien wesentlich gröBer sind, käme dies annähernd einer Einführung von Eurobonds gleich. Denn die Haushaltsrisiken der überschuldeten Staaten würden auf andere abgewälzt. Man wäre dann bei einer Haftungsgemeinschaft angelangt. Der größere Teil der Eurozone würde nicht mehr Transfers in einen deutlich kleineren Teil der Eurozone leisten, sondern Geber- und Nehmerstaaten würden sich in etwa die Waage halten - bei allmählicher Verwischung der haushaltspolitischen Verantwortlichkeiten. In einem solchen Szenario würden die fiskalische Kapazität und Toleranz der Geberstaaten überstrapaziert. In Verbindung beispielsweise mit einem hierdurch provozierten Rating-Verlust Frankreichs, dessen Triple-A-Rating jetzt schon auf der Kippe steht, könnte dies letztlich das Ende der Eurozone bedeuten. Die Europäische Zentralbank, deren Unabhängigkeit durch die Beschlüsse weiter in Zweifel gezogen wird, wäre nicht mehr ein Garant dafür, dass die EFSF die Option Anleihekauf nur im Ausnahmefall nutzt.

Der viel zu geringe Schuldenschnitt bedeutet unmittelbar, dass in Zukunft eine zusätzliche noch substanziellere Umschuldung notwendig wird, wenn es nicht noch einen höheren Transfer anderer Euro-Staaten an Griechenland geben wird. Es ist nicht ausgeschlossen, dass dies schon bald der Fall sein wird. Denn Schuldenschnitte wirken besonders, wenn sie 
überraschend kommen. Spätestens aber dürfte es eine substanzielle Umschuldung nach 2013 geben, wenn die EFSF durch den Europäischen Stabilitätsmechanismus (ESM) ersetzt wird und die Bundestagswahlen stattgefunden haben. Dies macht wegen des Vorhandenseins geeigneter institutioneller Rahmenbedingungen die Umstrukturierung privat gehaltener Staatspapiere einfacher. Schließlich dürften die präventiven Kreditlinien im Hinblick auf Italien das bisherige EFSF-Volumen deutlich überfordern, wiederum mit der Folge, dass es zu einer weiteren Umschuldung oder zusätzlichen Transfers kommen könnte.

\section{Kosten für den deutschen Steuerzahler}

Auch das zweite Rettungspaket für Griechenland hat die Entwicklung nicht stabilisiert. Vielmehr hat sich die Schuldenkrise nochmals verschärft, weil die Beschlüsse unzureichend sind und angesichts der tiefen Rezession in Griechenland keine Lösungsperspektive enthalten. Will man eine Transferunion vermeiden, gibt es für die Rettung Griechenlands nur eine Möglichkeit: einen möglichst frühzeitigen und kräftigen Schuldenschnitt. Alles andere hilft Griechenland nicht auf die Beine und riskiert die Zukunft des Euro, da eine unkontrollierte Insolvenz zu einem späteren Zeitpunkt immer noch droht. Dagegen liegt der Schuldenschnitt nach den Berechnungen der Gipfelteilnehmer nur bei 21 Prozent. Private Gläubiger werden nur auf freiwilliger Basis einbezogen. Man hofft, dass 90 Prozent der im Privatbesitz befindlichen Anleihen mit Fälligkeit zwischen 2011 und 2020 im Volumen von 150 Milliarden Euro in neue Anleihen umgetauscht werden.

Auf mehr konnte man sich nicht einigen, weil insbesondere die französischen Banken massive Verluste zu erwarten hätten. Diese sind in den Schuldenstaaten der Eurozone viel stärker engagiert als etwa die deutschen Finanzinstitute. Für 67 Milliarden Euro halten sie Staatsanleihen von Griechenland, Portugal, Irland, Spanien und Italien. Allein in Italien hat Frankreich für 32 Milliarden Euro Staatsanleihen im Feuer. Im Fall Griechenlands beträgt das Länderrisiko 46 Milliarden Euro. Damit könnte eine Umschuldung Griechenlands schnell gravierende Folgen haben und eine Kettenreaktion auslösen. Das Aufkaufen von Staatsanleihen der Schuldenstaaten und eine weitere Ausweitung der EFSF waren aus dieser Sicht die einzige Möglichkeit, Griechenland wenigstens ein wenig zu retten und selbst unbeschadet davonzukommen. Die EFSF kann indes nicht nur Kredite vergeben, sondern künftig Anleihen kaufen, um Spannungen aus dem Markt zu nehmen.

All dies erhöht das Risiko für die deutschen Steuerzahler erheblich. Dabei sind die Kosten davon abhängig, welche Annahmen bezüglich der künftigen Entwicklung konkret gemacht werden. Daneben können die Berechnungen der Kosten nur grobe Anhaltspunkte liefern. Dennoch verdeutlichen sie die hohe Belastung, die durch die Beschlüsse zu erwarten ist. Entsprechend der Annahmen schafft es Griechenland nicht wie geplant, Staatsbesitz in Höhe von 50 Milliarden Euro zu verkaufen, sondern nur für 25 Milliarden Euro. Angesichts des Unwillens, die Wirtschaft zu deregulieren, ist dies eine durchaus noch optimistische Einschätzung, die den künftigen Refinanzierungsbedarf des Staates jedoch weiter erhöht. Griechenland zahlt in allen Szenarien seine Zinsen. Die Rückzahlung der gewährten Kredite ist allerdings ungewiss.

In die Szenarien gehen die Altkredite an Griechenland, an denen Deutschland einen Anteil in Höhe von 10,4 Milliarden Euro hat, sowie die Kosten der Einrichtung des ESM, aus dem ab 2013 Kredite für notleidende Staaten bewilligt werden, ein. An der Bareinlage sind die deutschen Steuerzahler mit 22 Milliarden Euro beteiligt. Diese Summe kann als Versicherungsprämie interpretiert werden, um die Stabilität des Euro zu sichern. Sie wird annahmegemäß nicht zinsbringend angelegt. Denn Deutschland muss die Kosten durch Verschul- 
dung aufbringen, sodass sich hinsichtlich dieser Zinsströme ungefähr ein Nullsummenspiel ergeben würde. Neukredite, die ab 2011 gezahlt werden, orientieren sich am Refinanzierungsbedarf Griechenlands. Dabei werden allerdings Defizite innerhalb der Grenzen des Stabilitäts- und Wachstumspaktes hinzugezogen. Am Ende eines jeden Jahres erhalten die Gläubigerstaaten Zinserträge, die auf den Gegenwartswert diskontiert werden.

Selbst wenn Griechenland seine Kredite komplett zurückzahlt, bleibt bis 2020 eine Belastung für Deutschland von ungefähr 39 Milliarden Euro bestehen, unter anderem durch Bareinlagen beim Rettungsfonds ESM, dem beschlossenen Abschlag für griechische Verbindlichkeiten und Abschreibungen der Europäischen Zentralbank. So ist die Liquidität der griechischen Banken über das ELA-Kreditprogramm (Emergency Liquidity Assistance) der Notenbanken gesichert. Damit halten sich die hellenischen Geldhäuser über Wasser bis die Bonitätswächter das Rating wieder anheben und die Europäische Zentralbank ihre Staatspapiere uneingeschränkt als Sicherheit akzeptiert. Kann Griechenland seine Kredite nicht voll, sondern nur zu einem Drittel zurückzahlen, weil sich seine wirtschaftliche Lage kaum verbessert, erhöhen sich die deutschen Kosten auf gut 65 Milliarden Euro. Kollabiert der Staat trotz aller Hilfen und zahlt gar nichts zurück, müsste der hiesige Steuerzahler mit 79 Milliarden Euro geradestehen. Dabei sind dynamische Effekte, die beispielsweise aufgrund der Vertrauensverluste und der Ansteckung von Banken und anderen Staaten zu erwarten sind, nicht eingerechnet. Die Risiken könnten weiter steigen, denn die Peripheriestaaten hätten faktisch keinen Anreiz mehr, ihre maroden Haushalte zu sanieren. Sie würden den verhältnismäßig niedrigen Zinssatz im Euroraum nutzen und weiter Schulden anhäufen. Deutschland bliebe damit nichts anderes übrig, als regelmäßig neue Transfers zu leisten, solange die Währungsunion besteht. Und vielleicht ist ja sogar bald die nächste Hilfsaktion fällig. Der Präsident der Europäischen Kommission, José Manuel Barroso, fordert bereits, den mühsam ausgehandelten Rettungsschirm für Krisenstaaten zu erhöhen. ${ }^{6}$ Dass die Wirtschaft in Griechenland plötzlich erblüht, ist nicht zu vermuten, zumal von der Zinsseite durch die Entlastung noch weniger Anreize für eine rasche Wettbewerbsorientierung begründet werden.

\section{Stimulierung der Konjunktur in Griechenland}

Die Schuldenkrise in Griechenland hat sich seit den Beschlüssen des EU-Gipfels im Juli 2011 noch verschärft. ${ }^{7}$ Die wirtschaftliche Rezession, die seit Mitte 2007 anhält, verläuft deutlich tiefer als erwartet. Nach den letzten Prognosen wird für das Jahr 2011 mit einer Schrumpfung der Produktion gerechnet, die über 5 Prozent liegen dürfte. Schon in 2010 ist die Wertschöpfung um knapp 5 Prozent abgestürzt. Dabei haben sich vor allem die Perspektiven für den privaten Verbrauch, der mit mehr als 70 Prozent im EU-Vergleich einen hohen Anteil am Bruttoinlandsprodukt ausmacht, infolge von anhaltenden Einkommensverlusten und einer restriktiveren Kreditvergabepraxis der Banken verschlechtert. Die Arbeitslosigkeit hat sich in der Krise verdoppelt. Zudem wird die Kaufkraft der privaten Haushalte durch die höhere Inflation abgeschöpft. Dafür sind neben den weltweit anziehenden Energie- und Lebensmittelpreisen auch die Steuererhöhungen im Konsumbereich und gestiegene Abgaben verantwortlich, die die Regierung zur Konsolidierung der Staatsfinanzen beschlossen hat. Erst in 2012 könnte unter günstigen Bedingungen eine allmähliche Stabilisierung der Entwicklung erfolgen. Die Erholung wird jedoch durch die konjunkturelle Abkühlung in der

6 ZeitOnline: Barroso will mehr Geld für Euro-Rettungsfonds, 04.08.2011.

7 Rat der Europäischen Union: Erklärung der Staats- und Regierungschefs des Eurowährungsgebiets und der EU-Organe, Brüssel, den 21. Juli 2011. 
Weltwirtschaft gefährdet, die auch die Transportschifffahrt, eine der wichtigsten Branchen des Staates, beeinträchtigen wird. ${ }^{8}$

Die wirtschaftliche Entwicklung wird auch durch den harten Konsolidierungskurs bestimmt, mit dem sich die Regierung gegen die Schuldenkrise stemmt. Griechenland ist gegenwärtig mit 350 Milliarden Euro verschuldet. Die Schuldenquote, also der Schuldenstand gemessen am Bruttoinlandsprodukt, liegt bei mehr als 150 Prozent und das laufende fiskalische Defizit bei 10 Prozent. Die Märkte haben auf die Krise längst mit enormen Risikozuschlägen reagiert, die Ausfallprämien sind in kurzer Zeit extrem nach oben geschossen. Griechenland kann nur noch zu sehr hohen Zinsen frisches Geld am Kapitalmarkt erhalten. Nachdem Ratingagenturen die Kreditfähigkeit des Staates mehrfach herabgestuft hatten, hat die Troika im Frühjahr 2010 das erste Rettungspaket im Umfang von 100 Milliarden Euro mit einer Laufzeit von drei Jahren auf den Weg gebracht. Da sich eine Rückkehr an den Kapitalmarkt bis 2013 inzwischen als illusorisch herausgestellt hat, wurde das zweite Rettungspaket im Umfang von 109 Milliarden Euro vereinbart.

Griechenland erhält die (im Vergleich zum Kapitalmarkt) zinsverbilligten Kredite gegen strikte Einhaltung von Sparauflagen. Inzwischen konnten die Defizite in der Leistungsbilanz und im Staatshaushalt verringert werden. So sind die Lohnstückkosten gesunken, was die preisliche Wettbewerbsfähigkeit griechischer Produkte verbessert hat. Die Nettoexporte sind etwas gestiegen, obgleich dies nicht auf zunehmende Exportaktivitäten, sondern vor allem auf rückläufige Importe zurückzuführen ist. Die Reduktion der Lohnstückkosten reicht allerdings bei Weitem nicht aus, um die Fehlentwicklungen vergangener Jahre auszugleichen, in denen die Lohnzuwächse das Produktivitätswachstum übertrafen. So haben die Lohnstückkosten seit dem Beitritt des Staates zur Währungsunion im Jahr 2002 um 30 Prozent zugelegt, im Durchschnitt der Eurozone lediglich um 15 Prozent.

Die Rückführung der öffentlichen Budgetdefizite gestaltet sich schwieriger als erwartet, auch weil sich die Haushaltslage nach den Revisionen durch Eurostat als noch düsterer herausgestellt hat. Gleichwohl konnte das Defizit trotz einer schrumpfenden Wirtschaft um 5 Prozentpunkte verringert werden. Dazu wurde unter anderem die Mehrwertsteuer in mehreren Schritten bis auf 23 Prozent angehoben. Bei den Altersbezügen sind Kürzungen erfolgt, zum Beispiel durch Einführung einer Sonderabgabe auf Renten und ein höheres Renteneintrittsalter für Frauen. Im Gesundheitsbereich werden unter anderem durch die Senkung staatlich festgesetzter Preise für Medikamente Kosten gesenkt. Bei den Löhnen im öffentlichen Dienst, in dem 20 Prozent der Beschäftigten tätig sind, sind Zulagen gestrichen worden. Freie Stellen werden oft nicht mehr besetzt, befristete Stellen nicht mehr verlängert. Zuletzt ist eine Immobiliensteuer beschlossen worden, die zwei Milliarden Euro in die Kassen spülen soll. Um Möglichkeiten der Steuerumgehung einzuschränken, wird die Abgabe zusammen mit der Stromrechnung eingezogen. Zusätzlich sind Strukturreformen auf den Weg gebracht worden, um die Angebotsbedingungen zu stärken. Dazu zählen das Einfrieren von Mindestlöhnen, der Abbau von Überstundenzuschlägen, ein höherer Lohnverhandlungsspielraum auf betrieblicher Ebene und geringere bürokratische Auflagen bei der Gründung neuer Unternehmen. Das griechische Parlament hat Privatisierungen im Umfang von 50 Milliarden Euro beschlossen, die in den nächsten fünf Jahren angegangen werden. Diese sollen zum einen die Wettbewerbsfähigkeit der griechischen Wirtschaft stärken. Zum anderen wird der Staatshaushalt entlastet, weil es zu einem Schuldenabbau kommt. Daneben sind die

8 Vgl. beispielsweise Organisation for Economic Co-operation and Development (OECD): Greece at a glance: policies for a sustainable recovery, 2010, abrufbar unter: http://www.oecd.org/dataoecd/6/39/44785912.pdf (letzter Zugriff: 05.10.2011). 
Verbreiterung der Steuerbasis und die Eindämmung der Korruption erforderlich, um die Krise zu überwinden. ${ }^{9}$ Die Steuerschulden summieren sich inzwischen auf über 40 Milliarden Euro, davon werden 85 Prozent von 5 Prozent der Steuerflüchtigen geschuldet.

\section{Kurzfristige Wachstumsimpulse und Elemente einer Investitionshilfe}

Die Maßnahmen dürften allerdings erst in der längeren Frist ihre volle Wirkung entfalten. Kurzfristig führt die Konsolidierung zu einem massiven Einbruch des Bruttoinlandsprodukts, der eine rasche Erholung unwahrscheinlich macht. Zudem bleibt unklar, ob der Konsolidierungskurs auch über mehrere Jahre hinweg politisch durchgehalten werden kann. Eine möglichst rasche Beilegung der Schuldenkrise ist anzustreben, um ein Übergreifen auf andere Staaten zu verhindern und das Vertrauen in die Funktionsweise der Währungsunion zu stärken. Die Strategie immer neuer Kredite ist jedoch kaum nachhaltig und kann dazu führen, dass die Eurozone in eine Transferunion abgleitet, in der die Staaten mit gesunden Staatsfinanzen zahlen, um einen Bankrott der übrigen Staaten zu verhindern. ${ }^{10}$

Gleichwohl wird verschiedentlich eine deutliche Ausweitung der Transfers gefordert. Damit wären allerdings enorme Geldflüsse verbunden, die das Volumen der Rettungspakete um ein Vielfaches übersteigen. So haben Kai Konrad und Holger Zschäpitz errechnet, dass ein Ausbau nach Vorbild des deutschen Länderfinanzausgleichs jährliche Zahlungen in Höhe von rund 450 Milliarden Euro implizieren würde, wenn die Unterschiede in den Staatseinnahmen im Vergleich zum EU-Durchschnitt zur Hälfte nivelliert werden. ${ }^{11}$ Deutschland wäre mit 74 Milliarden, Frankreich mit 100 Milliarden Euro betroffen. Abgesehen davon, dass dies die Akzeptanz der Währungsunion infrage stellen würde, wäre ein solches System ohne eine finanzpolitische Integration nicht umzusetzen. So hätten die beteiligten Staaten ein Interesse an geringeren eigenen Steuereinnahmen. Behalten sie ihre finanzpolitische Hoheit, könnte ein Steuersenkungswettlauf ausgelöst werden. Eine Transferunion setzt daher die Harmonisierung der nationalen Steuersysteme voraus.

In der aktuellen Diskussion wird zunehmend gefordert, die Konsolidierungsprogramme durch kurzfristige Wachstumsimpulse für Griechenland zu ergänzen. Damit könnte der Konsolidierungskurs abgesichert werden, was nicht zuletzt auch im eigenen Interesse der Gläubigerstaaten liegt. So ist Deutschland aus griechischer Sicht seit Langem der wichtigste Handelspartner. Im letzten Jahr sind die Exporte nach Griechenland um 10 Prozent eingebrochen. Eine Stärkung der dortigen Entwicklung kann dazu beitragen, die Exportnachfrage zu stabilisieren und Arbeitsplätze in Deutschland zu sichern. Ein Investitionsförderprogramm gekoppelt mit den Privatisierungen könnte zudem den Umbau der griechischen Wirtschaft als Niedriglohnstandort in der Europäischen Union forcieren und die internationale Wettbewerbsfähigkeit auch der deutschen Wirtschaft erhöhen. Wenn man kostengünstig investieren kann und auf billige Arbeitskräfte stößt, rechnet sich Griechenland als Standort in der globalen Wertschöpfungskette. Daneben spielt Griechenland als Brückenkopf für den Seehandel zwischen Europa und Asien durch den Sueskanal eine Rolle. Damit könnte die starke Abhängigkeit von asiatischen Produktionsstandorten wie China vermindert werden.

In der Debatte wird verschiedentlich auf den Marshallplan als Vorbild verwiesen, der die wirtschaftliche Entwicklung im Nachkriegseuropa gefördert hat. Allerdings sollten die Wir-

9 Vgl. European Commission: The Economic Adjustment Programme for Greece, Fourth Review - Spring 2011, European Economy, Occasional Papers 82, Brüssel.

10 Vgl. Ansgar Belke/Christian Dreger: Ramifications of debt restructuring on the euro area - The example of large European economies' exposure to Greece, in: Intereconomics 4/2011, S. 188-196.

11 Vgl. Kai Konrad/Holger Zschäpitz: The future of the eurozone, 10.06.2011, abrufbar unter: http://www.voxeu.org/index.php?q=node/6628 (letzter Zugriff: 05.10.2011). 
kungen nicht überschätzt werden. So hat der Plan das Wirtschaftswachstum nach Berechnungen von Barry Eichengreen nur um 0,5 Prozent erhöht, die Hauptlast wurde von den einzelnen Staaten aus eigener Kraft geleistet. Die Wirtschaft ist in Deutschland, Österreich und Italien schneller gewachsen als in Großbritannien, Schweden und Griechenland, obwohl diese als frühere Alliierte größere Summen von den USA erhielten. Viel bedeutender für den deutschen Erfolg war die Erhard'sche Deregulierung von Preiskontrollen. Dies unterstreicht, dass die Unterstützung für Griechenland von ordnungspolitisch anreizkonformen Reformen begleitet werden muss, um die Hilfen effektiv werden zu lassen.

$\mathrm{Zu}$ den konkreten Hilfsmaßnahmen zählt eine Umleitung der EU-Finanzmittel. ${ }^{12}$ Dabei sollte es weniger um landwirtschaftliche Projekte und Infrastruktur, sondern um die Förderung kleiner und mittlerer innovativer Unternehmen und Businessparks gehen. Auch könnten Mittel verwendet werden, um die Kreditvergabe an innovationsfreudige kleine und mittlere Unternehmen zu erleichtern. Der Zugang zu Krediten ist derzeit für viele Unternehmen ein Hauptproblem. Dabei sind auch Firmen betroffen, die international konkurrenzfähig sind. Durch die Bonitätsabstufungen des Staates werden sie in Mithaftung genommen. Eine weniger restriktive Kreditvergabepraxis könnte die Realwirtschaft stimulieren. In diesem Bereich könnten auch Förderbanken ihr Engagement verstärken.

Des Weiteren sollte die Diskrepanz zwischen abgerufenen und bewilligten Mitteln aus den Struktur- und Kohäsionsfonds verringert werden. Der Abstand liegt für Griechenland bei 7 Prozent des Bruttoinlandsprodukts. ${ }^{13}$ Um Fördermittel zu erhalten, müssen Empfängerstaaten einen Eigenanteil leisten. Dies sichert einen sparsamen Umgang mit öffentlichen Geldern, kann aber in Zeiten fiskalischer Konsolidierung eine Wachstumsbremse darstellen. Daher könnte das Prinzip der Kofinanzierung temporär ausgesetzt werden. Dem Abruf der Mittel stehen auch administrative Hindernisse entgegen, etwa wenn wegen fehlender Katasterämter Eigentumsnachweise nicht vorgelegt werden können.

Daneben sind private Investitionen unter anderem in erneuerbare Energien durch einfache und transparente Genehmigungsverfahren zu forcieren. Zudem könnte die Projektfinanzierung der Europäischen Investitionsbank für Vorhaben im Bereich der Infrastruktur oder bei Hilfen für kleine und mittlere Unternehmen ausgedehnt werden. Allerdings sind auch hier Fehlanreize zu beachten, weil notleidende Staaten unterstützt werden, ohne dass es zu durchgreifenden Reformen kommt. Um zu gewährleisten, dass die Mittel tatsächlich wachstumsfördernd verwendet werden, werden eine Einschränkung der finanzpolitischen Souveränität Griechenlands und der Aufbau einer Treuhandanstalt nach deutschem Vorbild gefordert, die die anstehenden Privatisierungsaufgaben begleitet. Für Direktinvestitionen kommen viele Sektoren in Betracht. Hohe Potenziale bestehen vor allem bei der Informationstechnologie und erneuerbaren Energien, zumal die Arbeitnehmer in Griechenland sehr gut ausgebildet sind. Um die Investitionsanreize zu stärken, sind die Standortbedingungen zu verbessern und Genehmigungsverfahren zu vereinfachen und transparenter zu gestalten. Dabei könnte die Einrichtung von Sonderwirtschaftszonen mit zeitlich befristeten Steuersenkungen und Abschreibungserleichterungen die Investitionsanreize verbessern.

Insgesamt sind jedoch funktionierende Institutionen des Staates und der Wirtschaft unerlässlich. Die Fortsetzung von Strukturreformen sollte den Boden für stärkere Investitionsaktivitäten bereiten. Dieser Prozess kann von sequenziellen Hilfen begleitet sein, die an Re-

12 Vgl. Nikolaus Heinen: Transferunion Europa. Wie groß, wie stark, wie teuer?, Deutsche Bank Research: EUMonitor 81, 28.06.2011.

13 Vgl. Benedicta Marzinotto: A European fund for economic revival in crisis countries, Bruegel Policy Contribution 1/2011, Februar 2011. 
formschritte zu koppeln wären. Dies kann den Konflikt zwischen Konditionalität und Alternativlosigkeit auflösen und verhindert, dass Mittel in ineffiziente Strukturen fließen. Ansonsten besteht die Gefahr, dass der Anpassungsbedarf unterschätzt und Reformen verschleppt werden. Daher kann die Einrichtung einer europäischen Agentur mit Weisungskompetenz in einzelnen Politikfeldern unterstützend wirken.

\section{Zu wenig Spielraum, um unabhängig zu handeln. Die Europäische Zentralbank und ihre politischen Beschränkungen}

Wie hängt nun das griechische Schicksal mit demjenigen der Europäischen Zentralbank zusammen? Der erste Punkt ist der Folgende: Da Vertreter der Europäischen Zentralbank häufig argumentieren, dass sie quasi-fiskalische Tätigkeiten ausüben müssen, weil die Politiker ihre Hausaufgaben in Sachen finanzpolitischer Stabilität nicht erledigt hätten, ist es wichtig, die politische Ökonomie des (Nicht-)Handelns der Regierungen in der Eurozone näher zu betrachten. Die jüngsten Vorschläge zur Stärkung der Eurozone seitens der politischen Führung Deutschlands und Frankreichs haben umfangreiche Kritik, sogar von Schlüsselpersonen innerhalb der EU-Institutionen, dafür erfahren, ,zu wenig, zu spät“ zu bieten. ${ }^{14}$ Warum zögern die wichtigsten Akteure der Europäischen Union so lange damit, eine umfassende Lösung für die Euro-Schuldenkrise vorzulegen - ein Problem, das eine schwere und unmittelbare Hypothek für die Ära des designierten neuen Präsidenten der Europäischen Zentralbank Mario Draghi darstellt? ${ }^{15}$

Die Antwort ist sehr stark davon abhängig, wie gut das aktuelle Szenario, das im Folgenden genauer beschrieben wird, die Märkte und damit wohl auch die Regierungen der größeren Kredit-/Garantiegeber-Staaten treibt. Anfang August 2011 kam es zu einem Domino-Effekt und einem sprunghaften Wertverfall von Bank-Assets, weil die Finanzmärkte verständlicherweise nicht abwarteten, dass ein Staat nach dem anderen herabgestuft wird. Märkte neigen dazu, das ,Endspiel' oder zumindest ein mögliches Szenario daraus, das Schachmattsetzen der gesamten EFSF/ESM-Struktur zu antizipieren. ${ }^{16}$ Es kann als sicher gelten, dass Politiker sich dessen bewusst sind.

Die Märkte sahen sich zwischen drei recht inkonsistenten Zwängen gefangen, die von europäischen Politikern aufgrund der gegebenen Verhandlungsstruktur des ,Spiels ' nicht gelockert werden können. Zunächst ist eine beträchtliche Erhöhung der Kreditfähigkeit der EFSF technisch und politisch nicht möglich. Zweitens wird es in der kurzen bis mittleren Frist keine Eurobonds geben, weil diese gegen die Interessen des größten und (immer noch) solventesten Garantenstaats verstoßen. Und drittens ist die Europäische Zentralbank nur ungern in große Käufe finanzieller Risiken der nationalen Regierungen involviert und akzeptiert keine hinreichend großen ,haircuts' für überschuldete, das heißt insolvente Staaten wie Griechenland. Anders verhält sich dies in Bezug auf illiquide Staaten. Die deutsche Regierung zum Beispiel ist stark geneigt, die Europäische Zentralbank in dieser Hinsicht zu unterstützen. Denn der deutschen Bevölkerung wurden eine finanziell und politisch unabhängige

14 Martin Schulz, Vorsitzender der S\&D-Fraktion im Europäischen Parlament: Sozialdemokraten gegen MerkelSarkozy-Beschluss!, Pressemitteilung, 19.08.2011.

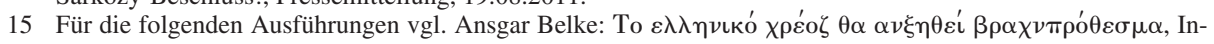
terview, in: The Sunday Eleftherotypia, 04.09.2011; Ansgar Belke: Change of guards in the ECB - Trichet's legacy in times of euro area and global imbalances, Briefing paper prepared for presentation at the Committee on Economic and Monetary Affairs of the European Parliament for the quarterly dialogue with the President of the European Central Bank, September 2011, Brüssel.

16 Vgl. Daniel Gros/Thomas Mayer: August 2011: What to do when the euro crisis reaches the core?, Centre for European Policy Studies: CEPS Commentaries, 11.08.2011. 
Europäische Zentralbank und eine strikt nationale Verantwortung der Mitgliedstaaten für ihre öffentlichen Finanzen im Austausch für die Aufgabe der DM versprochen.

Darüber hinaus ist die ausgefeilte Struktur des ,Endspiels‘ der Eurozone sehr schwierig zu handhaben. Unter anderem gibt es einen offensichtlichen Anreiz für schwächere Mitgliedstaaten zahlungsunfähig zu werden, solange noch genügend solvente Bürgen zur Verfügung stehen. Außerdem ist gegenwärtig, wie die Deauville-Einigung zwischen Angela Merkel und Nicolas Sarkozy im Oktober 2010 zeigte, die Zeit der , package deals`: In diesem Fall hat Deutschland sein Ziel der Härtung des präventiven Arms des Stabilitäts- und Wachstumspakts gegenüber Frankreich zum Teil geopfert, um von Frankreich die Zustimmung zur Einbeziehung des privaten Sektors in den ,haircut' zu erkaufen. Gefangen in einer Minderheitskoalition von Staaten mit soliden Finanzen (Finnland, Österreich und die Niederlande) ist Deutschland besonders erpicht auf diese Art von Absprachen. Denn der Staat fürchtet, am Ende in Vereinbarungen wie - etwa über eine ,echte' europäische Wirtschaftsregierung - von der Mehrheit der Euro-Staaten - den überschuldeten Staaten - gezwungen zu werden, ohne das Design dieser neuen Art der ,European economic governance' mit bestimmt oder gar dominiert zu haben.

Schließlich streben die Politiker natürlich danach, an ihrer ,Heimatbasis ‘ wiedergewählt zu werden. In Deutschland zum Beispiel werden die Bundestagswahlen im Jahr 2013 stattfinden, das heißt in dem Jahr, in dem der ESM eingerichtet werden soll. Unvergessen ist, dass Merkel wichtige Landtagswahlen wie in Nordrhein-Westfalen verloren hat, unmittelbar nachdem sie dem ersten griechischen Rettungspaket zugestimmt hatte.

Folglich hat das häufig als zögerlich und halbherzig bezeichnete Verhalten der Politiker womöglich ein klares Motiv. Ökonomen würden sagen: Euro-Staaten maximieren ihren Macht-Index, das heißt die Wahrscheinlichkeit der Mitgliedschaft in gewinnbringenden Koalitionen. ${ }^{17}$ Natürlich bedeutet diese polit-ökonomische Konstellation eine große Herausforderung für einen weiteren wichtigen Spieler: die Europäische Zentralbank. Daher kann es als eine große Errungenschaft aus der Sicht der Europäischen Zentralbank gelten, dass JeanClaude Trichet aus den Verhandlungen auf dem EU-Gipfel am 21. Juli 2011 mit einem erwarteten Nettoverlust von Null herauskam. Denn jeder potenzielle Bewertungsverlust der Europäischen Zentralbank durch ,haircuts' wurde durch Vollgarantien kompensiert, bereitgestellt vom europäischen Steuerzahler.

Ein zweiter entscheidender Punkt geht von der Beobachtung aus, dass die Forcierung der EFSF von vielen führenden Ökonomen und Marktanalysten als ein notwendiger Schritt zur Beruhigung der Märkte betrachtet wird.

Zunächst einmal sollte man im Hinterkopf behalten, dass die EFSF ursprünglich für eine Krise in der Peripherie entwickelt wurde. Große Euro-Staaten bieten also im Notfall ausschließlich kleinen Staaten wie Griechenland, Irland und Portugal finanzielle Unterstützung. Insbesondere hat die EFSF einfach nicht genügend Ressourcen, um die massiven Käufe von Anleihen zu tätigen, die erforderlich werden, um die Märkte zu stabilisieren.

Darüber hinaus machen die Regeln der EFSF diese anfällig für einen Domino-Effekt: Einem Staat, der durch finanzielle Schwierigkeiten geplagt wird und selbst Unterstützung von der EFSF einfordert, ist es erlaubt auszutreten. ${ }^{18}$ Nach Art. 2 Abs. 7 des EFSF-Rahmenver-

17 Vgl. Ansgar Belke/Barbara Styczynska: The Allocation of Power in the Enlarged ECB Governing Council: An Assessment of the ECB Rotation Model, in: Journal of Common Market Studies 5/2006, S. 865-897; Ansgar Belke/Barbara von Schnurbein: European monetary policy and the ECB rotation model - Voting power of the core versus the periphery, erscheint in Public Choice.

18 Vgl. Daniel Gros/Alessandro Giovannini: The EFSF as European Monetary Fund: Does it have enough resources?, Centre for European Policy Studies: CEPS Commentaries, 22.07.2011. 
trags ${ }^{19}$ kann er somit keine Garantien für eine weitere Schuldenaufnahme durch die EFSF übernehmen. Darüber hinaus wird ein Staat mit hohen Kosten der Kapitalbeschaffung (wie im Fall von Italien und Spanien, wenn die Zinsen auf ihrem zu hohen Krisen-Niveau verbleiben) als Bürge aussteigen und nur der Kern der Eurozone bliebe als Bürge der EFSF zurück. An diesem Punkt würde die auf den Kernstaaten ruhende Belastung unerträglich werden - wegen der gerade abgeleiteten Kaskaden-Struktur der EFSF. ${ }^{20}$

Daher sind Politiker und auch Vertreter der Europäischen Zentralbank wie Draghi gut beraten, anzuerkennen, dass es erhebliche Gefahren bei der Anwendung der Peripherie-Lösung für den Kern gibt. Dies macht sofort verständlich, dass - unter den gegenwärtigen institutionellen Gegebenheiten - eine immer größere EFSF nicht die Lösung sein kann. Im Gegenteil, jede beabsichtigte Erhöhung um Beträge von 2,5 Billionen Euro bis zu schier unglaublichen 4 Billionen Euro könnte den Domino-Effekt noch beschleunigen, da dies entweder zu, stepouts ' einer wachsenden Zahl von Bürgen oder zu ständig steigenden Refinanzierungskosten der EFSF führen würde, wenn diese Staaten wegen des mit einem Ausstieg verbundenen Reputationsverlustes in der EFSF verblieben. ${ }^{21}$

Dass auch die französische Regierung ihr Interesse an einer Ausweitung der EFSF verringert hat, macht aus französischer Perspektive viel Sinn. Sarkozy musste erkennen, dass die Finanzmärkte das Domino-Risiko verstanden und tatsächlich angefangen haben, auch die französischen Fremdkapitalkosten in die Höhe zu treiben. Bloße Gerüchte über eine Ausweitung der EFSF haben Frankreich zu dem Kernland der Wirtschafts- und Währungsunion gemacht, das der größten Gefahr des Verlustes seines AAA-Ratings ausgesetzt ist. Aber wenn Frankreich seinen AAA-Status wirklich verlieren sollte und dann gezwungen wäre, aus der EFSF auszusteigen, verbliebe nur Deutschland (und einige seiner kleineren Nachbarn wie Österreich, die Niederlande und Finnland, die im gegenwärtig stattfindenden polit-ökonomischen Spiel als Deutschlands Verbündete bezeichnet werden können), um die gesamte Last zu tragen. Dies wäre nicht nur politisch inakzeptabel und würde deshalb zu wachsenden nationalistischen Tendenzen führen, sondern es läge auch wirtschaftlich außer Reichweite. Denn in Bezug auf das Bruttoinlandsprodukt (BIP) ist Italien achtmal Griechenland und die gesamten Staatsschulden Italiens entsprechen fast dem gesamten deutschen BIP.

Schließlich haben wir häufiger schon an anderer Stelle argumentiert, dass eine Ausweitung der EFSF der Einführung einer Variante der ,Euro-Anleihen“ entspricht, da die Mitgliedstaaten gemeinsam für jeden anderen garantieren. ${ }^{22}$ Dies steht in starkem Kontrast zu dem Rettungsprogramm für die Peripherie, das durch einen einseitigen Fluss von Garantien und Mitteln geringerer Größenordnung gekennzeichnet ist. Allerdings würde jeder ,Euro-Anleihe`Ansatz eine Änderung der EU-Verträge nötig machen. Schließlich wäre diese wahrscheinlich nicht mit der deutschen Verfassung kompatibel, was zumindest für die deutsche Regierung relevant ist und deren Verhalten als Spieler im aktuellen ,Euro-Endspiel` beeinflusst.

19 EFSF Rahmenvertrag zwischen Königreich Belgien, Bundesrepublik Deutschland, Irland, Königreich Spanien, Französische Republik, Italienische Republik, Republik Zypern, Großherzogtum Luxemburg, Republik Malta, Königreich der Niederlande, Republik Österreich, Portugiesische Republik, Republik Slowenien, Slowakische Republik, Republik Finnland, Hellenische Republik und European Financial Stability Facility, 7. Juni 2010.

20 European Financial Stability Facility: European Financial Stability Facility (EFSF), 02.08.2011, abrufbar unter: http://www.efsf.europa.eu/attachments/faq_en.pdf (letzter Zugriff: 05.10.2011).

21 Vgl. Jürgen Michels/Ebrahim Rahbari/Willem H. Buiter/Giada Giani: Global economics view: Buying time for the euro: A proposal, in: Willem Buiter/Michael Saunders/Kiichi Murashima/Robert V. DiClemente/David Lubin/Johanna Chua/Joaquin A. Cottani: Citigroup Global Markets, 20.07.2011, S. 16-23; und Gros/Giovannini: The EFSF as European Monetary Fund, 2011.

22 Der Begriff ,Euro-Anleihen“ wird in der öffentlichen Debatte häufig nicht konkret genug verwendet. 
Ein dritter Punkt geht von der Beobachtung aus, dass Griechenland ein nicht mehr handhabbares Niveau der Verschuldung aufweist, das scheinbar jeder - mit Ausnahme der EUFührung - kennt. In der Tat scheint kein bisher entwickelter Plan das griechische Schuldenproblem adäquat zu adressieren. So obliegt der Europäischen Zentralbank die wichtige Herausforderung, konkret anzugeben, unter welchen Bedingungen Griechenland seine Insolvenz vermeiden und in der Eurozone bleiben kann - ganz ähnlich wie sie der italienischen Regierung im Spätsommer Inhalte des jüngsten Sparprogramm diktierte. Sonst wäre Italien nicht in den Genuss der Staatsanleihenkäufe durch die Europäische Zentralbank gekommen.

Besorgniserregend ist, dass unter den derzeitigen Prognosen die griechischen Schulden nach dem Gipfel vom 21. Juli 2011 kurzfristig noch angestiegen sind und in den kommenden Jahren auf einem Niveau von über 100 Prozent des BIP verharren werden. Gerade deshalb sehen wir die Notwendigkeit einer weiteren Anpassung des , haircuts ' bereits in der näheren Zukunft.

Bereits zuvor wurde ausgeführt, dass das Ausmaß der konkret beschlossenen Maßnahmen enttäuschend ist, obwohl Teile der Vereinbarungen des jüngsten Gipfeltreffens vom Juli 2011 in die richtige Richtung weisen. Es hat nicht das Potenzial, ein Ende der Schuldenkrise herbeizuführen. Stattdessen wird das Risiko der Ansteckung anderer Staaten noch erhöht. Dies liegt daran, dass ein viel zu niedriger , haircut‘ sofort das Signal an die Marktteilnehmer sendet, dass eine noch erheblichere Umstrukturierung, wenn nicht sogar ein gigantischer Geldtransfer von Euro-Staaten nach Griechenland erforderlich sein wird. Vermutlich werden wir eine noch erheblichere Umstrukturierung der Schulden in der Zeit nach 2013 sehen, wenn die EFSF durch den ESM ersetzt wird und die deutschen Wahlen stattgefunden haben. Diese Ansicht wird in technischer Hinsicht durch die Tatsache gestützt, dass die Existenz des ESM die Umstrukturierung von privat gehaltenen Staatsanleihen viel einfacher macht.

So wird auch die Europäische Zentralbank unter der Leitung von Draghi gefordert sein, ihren Teil zu dem Prozess der Definition von Bedingungen für eine nachhaltige Lösung im Rahmen der gesamten Eurozone beizutragen. Dieser Prozess könnte wie folgt aussehen, ist aber immer noch eine Aufgabe für künftige Bemühungen. Letztere aber werden von enormer Bedeutung für die künftige Rolle der Europäischen Zentralbank sein.

Lassen Sie uns von der gängigen Beobachtung starten, dass (nicht nur) die griechischen Banken das schwächste Glied in der Kette sind. Sie beschleunigen die Übertragung des oben beschriebenen Domino-Effekts. Denn viele Banken halten große Bestände an Staatsschuldpapieren in ihren Büchern. Zudem schrumpft ihre Bonität dabei parallel zu derjenigen ihrer Standorte. Dies genau ist wohl immer das Motiv für Trichet gewesen, so sehr auf die Entkoppelung der Banken der Eurozone vom Schicksal ihrer Volkswirtschaften aus zu sein.

Es ist nun offensichtlich, dass die Kapitalmärkte das Potenzial eines ,Doomsday '-Szenarios antizipieren, in dem die Volkswirtschaften abrupt in eine Rezession fallen, da der Interbankenmarkt zusammenbricht und die öffentliche Verschuldung weiter wächst. Leider werden sich diese Erwartungen materialisieren, solange der Zusammenbruch des Interbankenmarktes nicht unmittelbar adressiert wird. ${ }^{23}$ Um nur die schlimmsten denkbaren Szenarien zu umgehen, wäre es nach Daniel Gros und Thomas Mayer eine Option, zu berücksichtigen, dass die Eurozone eine groß angelegte Infusion von Liquidität benötigt ob man nun bereit ist, dies zu akzeptieren oder nicht. Da die bestehende Kaskaden-Struktur der EFSF ein Teil des Problems ist, kann die Lösung nicht in einem massiven Anstieg des Umfangs der EFSF bestehen. Vielmehr sollte gemäß Gros und Mayer die EFSF Zugang zur

23 Vgl. Gros/Mayer: What to do when, 2011. 
Refinanzierung bei der Europäischen Zentralbank als Lender-of-last-resort (,Gläubiger der letzten Instanz') erhalten. ${ }^{24}$ Die EFSF würde damit endgültig in einen Europäischen Währungsfonds (EMF) umgewandelt werden.

Mit Blick auf die Struktur des polit-ökonomischen ,Euro-Spiels‘ ist zu vermuten, dass Deutschland jede Lösung über die EFSF am Ende jeder Lösung vorziehen wird, die der Kommission eine prominente Rolle zuweist. Denn dieser Staat hegt zunehmend Zweifel an dem nicht-politischen Charakter der Europäischen Kommission mit ihrer angeblichen Verzerrung der Präferenzen zugunsten der überschuldeten Staaten des Südens wie Portugal und Italien.

Wenn auch die Europäische Zentralbank unter Draghi bereit wäre, dem Ansatz von Gros und Mayer zu folgen, ${ }^{25}$ müsste Deutschland mit seiner regelbasierten und auf Preisstabilität bedachten Bundesbank-Tradition folgen und die bittere Pille schlucken, dass diese Änderung im Charakter der Europäischen Zentralbank der Preis für die nachhaltige Durchsetzung des einzigen Wegs ist, um die derzeitigen Probleme zu überwinden: Die Regierungen der hoch verschuldeten Euro-Staaten sollten weiterhin bereit sein, die Krise des Vertrauens an den Märkten durch eine aktive und nachhaltige Verbesserung ihrer Wettbewerbsfähigkeit und ihrer Wachstumsperspektiven an ihren Wurzeln zu packen.

\section{Von der Überschuldung zum Währungswettbewerb}

Will man die Europäische Währungsunion wieder nachhaltig auf die Spur setzen, müssen die Regierungen Schulden und Defizite erheblich reduzieren - optimalerweise durch demokratische durch die nationalen Parlamente beschlossene Schuldenbremsen. Die jüngste Finanz- und Wirtschaftskrise hat einmal mehr gezeigt, dass jedes Konzept des permanenten ,deficit spending' und der Überschuldung zwangsläufig zu der Unmöglichkeit des Schuldenablösens führt. Ein langfristig ausgerichtetes Programm des Schuldenabbaus ist hierfür eine conditio sine qua non. Allerdings benötigt der Schuldenabbau nicht nur in Griechenland Zeit. Um das Scheitern des Projekts der Wirtschafts- und Währungsunion zu vermeiden, sollte dies durch einen Krisenmanagement-Mechanismus wie den oben beschriebenen begleitet werden, der - wie von Draghi immer wieder zu Recht betont ${ }^{26}$ - noch nicht von ,Euro-Anleihen' begleitet werden sollte. Eine wesentliche Voraussetzung für die Einführung von ,Euro-Anleihen“ in einer engen Definition ist, dass die öffentliche Verschuldung zunächst auf ein nachhaltiges Niveau reduziert worden ist. Aber die Einhaltung dieser entscheidenden Bedingung ist in der Eurozone weder in Sichtweite, noch glaubhaft auf der Tagesordnung, zumindest vorerst. Stattdessen hat man sie in Deauville im Oktober 2010 weiter verwässert.

Insgesamt gesehen ist wohl nicht sicher, dass der Vorschlag von Gros und Mayer eine ,Gleichgewichts-Lösung' im zuvor beschriebenen polit-ökonomischen ,Spiel` darstellen kann, ${ }^{27}$ da er gegen die kurzfristigen Interessen der deutschen Regierung durchgesetzt werden müsste. Allerdings ist es das Verdienst dieses Vorschlags, ohne Umwege zur direkten Konfrontation mit schmerzhaften, aber höchst relevanten Herausforderungen zu führen:

24 Ebenda. Bei einem Lender-of-last-resort handelt es sich um eine Institution, die freiwillig oder auf gesetzlicher Grundlage als Kreditgeber oder Garant bei Schuldnern fungiert, wenn hierzu keine andere Institution mehr bereit ist. Auf supranationaler Ebene übernimmt der Internationale Währungsfonds häufig diese Funktion. Auf Ebene des nationalen Bankwesens ist es zumeist die Zentralbank.

25 Ebenda.

26 Vgl. Mario Draghi: Alle sollten dem Beispiel Deutschlands folgen, Interview, in: Frankfurter Allgemeine Zeitung, 15.02.2011.

27 Vgl. Gros/Mayer: What to do when, 2011. 
(1) die Einführung glaubwürdiger Selbstverpflichtungen aller Regierungen in der Eurozone, um ein chronisches, deficit spending " ein für allemal zu verhindern, sowie eine gezielte Einführung wasserdichter Schuldenbremsen sind wahrscheinlich nicht mehr als eine Fiktion und (2) die Erkenntnis, dass - wenn man diesen Vorschlag ablehnt, weil er sehr nah an einer direkten monetären Finanzierung öffentlicher Schulden liegt - die einzige offensichtliche Alternative ein Währungswettbewerb oder sogar das Ende des Papiergeld-Standards (,fiat money“ $)^{28}$ selbst wäre. ${ }^{29}$ Die erste Alternative widerspräche nicht zuletzt der Notwendigkeit einer stärkeren Koordinierung der Geldpolitiken zwischen den G20. ${ }^{30}$

Eine ultimative Lösung bestünde darin, den Prozess der Geldschöpfung im Sinne der Österreichischen Schule wieder zu re-nationalisieren und zu entpolitisieren. ${ }^{31}$ Realistischerweise würde dies für Draghi zumindest bedeuten, die Europäische Zentralbank wieder zu befähigen, um den Ruf der Eurozone als stabilitätsorientiertesten Währungsraum der Welt konkurrieren zu können. Das Ziel dabei sollte sein, das Vertrauen der internationalen Investoren aus China, Russland und den erdölexportierenden Staaten zu gewinnen, die derzeit kurz davor stehen, den USA als sicheren Hafen aufgrund ihres offensichtlich nicht nachhaltigen makroökonomischen Politik-Mix den Rücken zuzukehren. ${ }^{32}$ Dies würde für Draghi unmittelbar die Notwendigkeit bedeuten, den Ausstieg aus der expansiven unkonventionellen Geldpolitik notfalls auch im Rahmen eines Alleingangs zu beschleunigen und die Kürzung der Bilanzen der Europäischen Zentralbank so schnell wie möglich voranzutreiben.

28 Jedenfalls dürften nicht nur monetaristische Puristen argumentieren, dass auch alle Alternativen wie eine Beteiligung des öffentlichen Sektors (wie das ,private sector involvement', PSI) im Endeffekt genau das Gleiche bedeuten: eine Finanzierung öffentlicher Verschuldung durch die Notenpresse. PSI impliziert nämlich, dass der private Sektor in dem einen oder anderen der Staaten von diesen gerettet wird, die dann wiederum von der Europäischen Zentralbank durch Anleihekäufe aufgefangen werden.

Ob der Vorschlag von Gros und Mayer tendenziell zu höherer Inflation führt, ist wohl noch eine offene Frage. Die Autoren argumentieren, dass die Geldnachfrage der EFSF einfach die Geldnachfrage des privaten Sektors verdrängt. Vgl. Gros/Mayer: What to do when, 2011.

29 Vgl. Ansgar Belke/Thorsten Polleit: Monetary economics in globalised financial markets, Berlin/Heidelberg, 2010.

30 Vgl. Ansgar Belke/Kerstin Bernoth/Ferdinand Fichtner: Die Zukunft des internationalen Währungssystems, Gutachten für das Bundesministerium der Finanzen, Deutsches Institut für Wirtschaftsforschung (DIW), Berlin 2011.

31 Für einen Vergleich des Friedman'schen konstanten Geldmengenwachstumsplans mit Hayeks Konzept des Währungswettbewerbs siehe Ansgar Belke/Thorsten Polleit: Monetary economics, 2010, S. 352-353.

32 Vgl. Ansgar Belke/Thorsten Polleit: How much fiscal backing must the ECB have? The euro area is not the Philippines, in: Économie Internationale 4/2010, S. 5-30. 